(2) Open Access Full Text Article

\title{
Cystoid Macular Edema Following Cataract Surgery with Low-Energy Femtosecond Laser versus Conventional Phacoemulsification
}

This article was published in the following Dove Press journal:

Clinical Ophthalmology

\author{
Stefaan Van Nuffel ${ }^{1} * *$ \\ Matthias F Claeys ${ }^{1, *}$ \\ Marnix $\mathrm{H}$ Claeys $^{2}$ \\ 'Department of Ophthalmology, \\ University Hospital Ghent, Ghent, \\ Belgium; ${ }^{2}$ Okulus Eye Clinic, Kortrijk, \\ Belgium \\ *These authors contributed equally to \\ this work
}

Purpose: To compare postoperative changes in central subfield macular thickness (CSMT) and prevalence of cystoid macular edema (CME) in patients undergoing cataract surgery with low-energy femtosecond laser versus standard phacoemulsification.

Design: This was a retrospective comparative real-world study.

Methods: Postoperative data of 252 eyes of 165 patients were collected: 138 eyes received low-energy femtosecond laser-assisted cataract surgery (FLACS) and 114 eyes underwent conventional phacoemulsification cataract surgery (CPCS). Postoperative changes in CSMT and prevalence of CME were compared between the two groups.

Results: There was a significant increase in mean CSMT from preoperative to postoperative values at 2.5 months in both the FLACS and CPCS group $(p<0.001)$. Mean change (preoperation to 2.5 months postoperation) in CSMT was $6.2 \pm 11.5 \mu \mathrm{m}$ in the FLACS group and $7.3 \pm 26.6 \mu \mathrm{m}$ in the CPCS group, which was statistically significant but clinically not relevant. Comparison of mean changes in CSMT (preoperation to 2.5 months postoperation) between the FLACS and CPCS groups revealed no significant differences. The rate of pseudophakic CME (PCME) development was lower in the FLACS group (1.4\%) than the CPCS group $(4.4 \% ; p=0.247)$. When using PREMED criteria to define clinically significant PCME, only $0.7 \%$ patients in the FLACS group and $1.8 \%$ in the CPCS group showed development of clinically significant PCME $(p=0.586)$.

Conclusion: The mean change in CSMT and occurrence of postoperative CME was lower in the low-energy FLACS group than the standard-phacoemulsification group; however, the difference was not statistically significant.

Keywords: cystoid macular edema, low-energy femtosecond laser, femtosecond laserassisted cataract surgery, phacoemulsification cataract surgery

\section{Introduction}

Phacoemulsification is the standard of care for the surgical removal of cataracts. Advancements in surgical techniques, biometry, IOL power-calculation methods, and IOL technology have contributed significantly to achieving excellent visual and refractive outcomes after cataract surgery. Despite such advancements, development of pseudophakic cystoid macular edema (PCME) in some cases is the most common cause of unexpected vision loss after uneventful surgery in patients without risk factors. ${ }^{1-5}$

Although the pathogenesis of PCME is not fully understood, it is mainly attributed to intraocular inflammation arising from surgical tissue manipulation and thermal damage due to the heat generated from the ultrasonic waves during
Correspondence: Stefaan Van Nuffel Email stefaan.vannuffel@hotmail.com 
phacoemulsification. ${ }^{6}$ The introduction of the femtosecond laser (FL) to create corneal incisions, capsulorhexis, and perform nuclear fragmentation has contributed to a reduction in ultrasound energy and time required for phacoemulsification, with corresponding reductions in postoperative inflammation, ${ }^{7-12}$ as is evident from the reduction in anterior-chamber flare following FL-assisted cataract surgery (FLACS). ${ }^{4,13-15}$ As such, it was expected that laser pretreatment may potentially reduce the risk of postoperative PCME.

There is contrasting evidence on the potential risk of developing PCME after FLACS relative to conventional phacoemulsification cataract surgery (CPCS). On one hand there have been reports documenting no significant differences in the rate of PCME development between FLACS and CPCS, ${ }^{14-21}$ but others have reported a trend toward higher PCME rates after FLACS compared with CPCS. ${ }^{2,22}$ In addition to the inflammation caused by phacoemulsification, higher PCME rates following FLACS have been attributed to inflammation resulting from laser pretreatment itself.

Laser-pulse energy has been associated with the degree of inflammatory response generated by FLs. ${ }^{19,23-25}$ Studies assessing the rate of PCME development in FLACS have employed high-energy laser platforms that emit high-energy (microjoule) coupled with low-frequency $(\mathrm{kHz})$ pulses. Laser pretreatment with high-energy FLs has been hypothesized to induce light toxicity, cause mechanical trauma due to microbubble formation, and thermal tissue damage due to vibrations or shockwaves, leading to increased prostaglandin release, which in turn is associated with increased pupillary miosis during FLACS. ${ }^{2,26,27}$ In contrast, low-energy FLs that emit energy in the nanojoule range and have a high pulserepetition rate (above $1 \mathrm{MHz}$ ) have been associated with less collateral damage to the surrounding ocular tissue and low release of prostaglandins. ${ }^{28}$ In this study, we investigated the risk of postoperative PCME development following FLACS with a low-energy FL compared with CPCS.

\section{Methods}

This retrospective comparative pilot study analyzed the data of 252 eyes of 165 patients who had undergone FLACS with the low-energy Femto LDV Z8 laser platform (Ziemer Ophthalmic Systems, Port, Switzerland) or CPCS in a real world setting between 01/10/2015 to 20/10/ 2018 at the Okulus Eye Clinic (Kortrijk, Belgium). Patients were counseled about the two treatment modalities, their pros and cons and costs involved, and offered both modalities of surgery. Written informed consent for undergoing surgery was obtained from all patients based on their choice of surgical modality. A total of 138 consecutive eyes chosen for FLACS and 114 consecutive eyes chosen for CPCS were included.

Deidentified data of patients were collected and analyzed in accordance with the tenets of the Declaration of Helsinki and its amendments. The study was approved by the Commission for Medical Ethics of Okulus Eye Clinic on September 25, 2015. All patients underwent preoperative optical coherence tomography (OCT imaging) to check for retinal pathology. In the CPCS group, one patient had a history of a branch retinal venous occlusion and four had an epiretinal membrane. These patients were not excluded. One patient was excluded because of a macular hole. In the FLACS group, one patient had an epiretinal membrane and was not excluded.

\section{Surgical Technique}

The Infiniti system (Alcon Laboratories) was used for phacoemulsification. In the CPCS group, a clear corneal incision of $2.2 \mathrm{~mm}$ was made. In the FLACS group, the Femto LDV Z8 system was employed for the creation of a $2.2 \mathrm{~mm}$ incision, capsulorhexis, and lens-fragmentation (an eight-piece pie-cut pattern). All surgeries were performed by the same surgeon (MC). None of the patients received topical nonsteroidal anti-inflammatory (NSAIDs) preoperatively. Dilation on the day of surgery was done with tropicamide, phenylephrine, and cyclopentolate drops. Postoperatively, patients were prescribed combination drops of tobramycin $3 \mathrm{mg} / \mathrm{mL}$ and Dexamethasone $1 \mathrm{mg} / \mathrm{mL}$ three times per day and ketorolac $5 \mathrm{mg} / \mathrm{mL}$ drops three times per day. This regimen was applied for 4 weeks in nondiabetic patients and 6 weeks in diabetic patients.

\section{OCT Measurements and Study Variables}

Macular spectral-domain OCT was performed on undilated eyes with a modular ophthalmic imaging platform (Spectralis, Heidelberg Engineering) and central subfield macular thickness (CSMT) extracted from the software. Measurements were taken preoperatively, on postoperative day 1 and at 2.5 months ( \pm 15 days) postoperatively. The CSMT was defined as the mean of the measurement in the central $1 \mathrm{~mm}$ area around the foveal centre. Presence of CME was assessed using two criteria: OCT imaging - appearance of cystoid changes near fovea; and PREMED study criteria - clinically significant CME, defined as occurrence of cystoid changes near the fovea accompanied with a change in CSMT of $10 \%$ or more and 
lower-than-expected best-corrected visual acuity on one or more postoperative controls.

\section{Statistics}

Statistical analyses were performed with SPSS version 26.0 (IBM, Armonk, NY, USA). Continuous variables were expressed as means \pm standard deviation. Categorical data were analyzed using $\chi^{2}$ tests and Fisher's exact test in cases where assumptions of the $\chi^{2}$ test were not met. Normality of the scale data was checked with the Shapiro-Wilk test. For normally distributed data, Student's $t$-test for paired data was used for comparisons between preoperative and postoperative data and the Wilcoxon signed-rank test to assess the significance of such differences when data were not normally distributed. For comparing means between the two groups, independent-sample $t$-tests or their nonparametric counterpart Mann-Whitney $U$ was used. Differences were considered statistically significant at $p<0.05$.

\section{Results}

There was no significant difference in mean keratometry, spherical equivalent, or axial length between the FLACS and the CPCS groups. Mean age was significantly different between the two groups (FLACS 68.73 vs CPCS 75.43 years, $p<0.001)$. In patients receiving FLACS, there was a significant increase in mean CSMT from preoperatively to 2.5 months postoperatively ( $p<0.001$ ) (Figure 1$)$. A similar trend was observed in the CPCS group $(p<0.001$, Figure 1); however, the increases of $6.2 \pm 11.5 \mu \mathrm{m}$ in the FLACS group and $7.3 \pm 26.6 \mu \mathrm{m}$ in the CPCS group were not clinically relevant. Comparison of mean changes in CSMT (from preoperatively) between the FLACS and the CPCS group

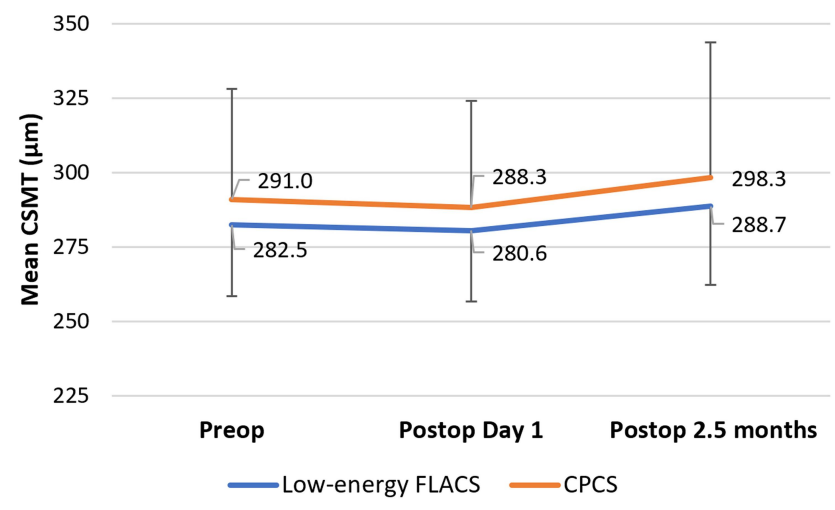

Figure I In patients receiving FLACS, there was a significant increase in mean central subfield macular thickness from preoperation to 2.5 months postoperation (blue line, $p<0.00 \mathrm{I}$ ). A similar trend was observed in the CPCS group (orange line, $p<0.001)$.

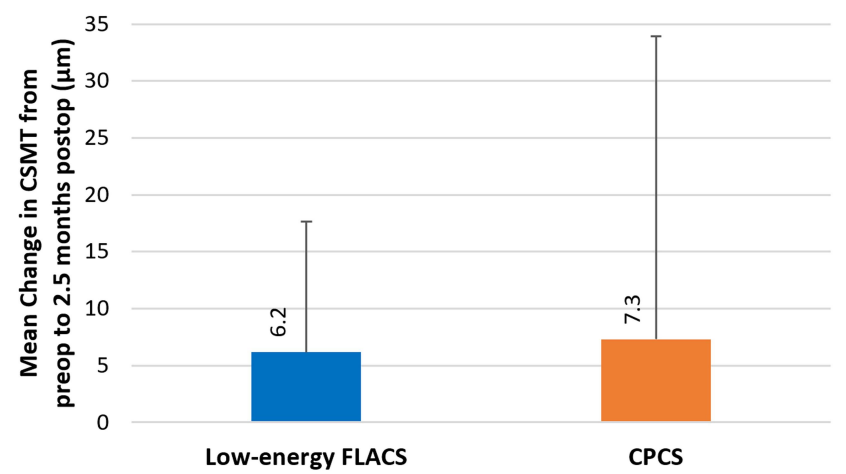

Figure 2 Comparison of mean changes in central subfield macular thickness (preoperative) between the FLACS (blue histogram) and CPCS groups (orange histogram) revealed no significant differences at day 1 or at 2.5 months postoperatively.

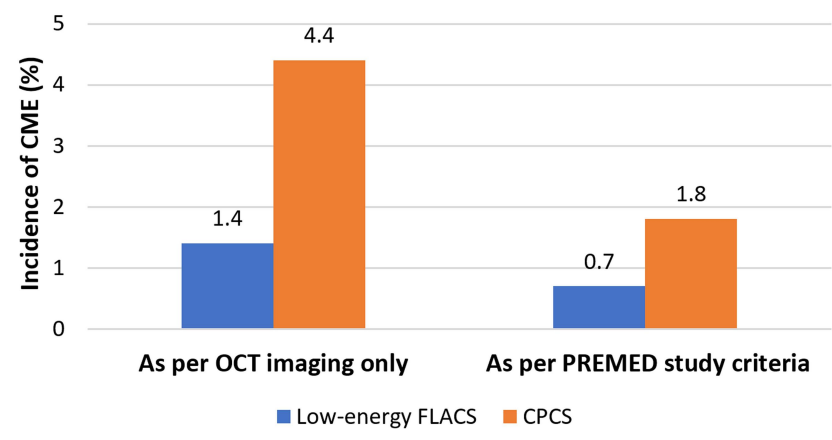

Figure 3 On the left, the rate of postoperative cystoid macular edema development is depicted as per OCT imaging only for the FLACS (blue histogram) and CPCS groups (orange histogram). On the right, the rate of postoperative cystoid macular edema development is depicted as per the PREMED criteria, for the FLACS (blue histogram) and CPCS groups (orange histogram).

revealed no significant difference at day $1(p=0.135)$ or 2.5 months postoperatively ( $p=0.902$, Figure 2 ).

The rate of PCME development was 3\% lower in the low-energy FLACS group than the CPCS group $(1.4 \%$ vs $4.4 \%$ ), although the difference did not reach statistical significance ( $p=0.247$, Figure 3 ). When PREMED criteria were used to define clinically significant PCME, only $0.7 \%$ patients in the FLACS group and $1.8 \%$ patients in the CPCS group showed the development of clinically significant PCME. Although the risk of developing clinically significant PCME was $1.1 \%$ lower in the low-energy FLACS group than the CPCS group, the difference was not statistically significant $(p=0.586)$.

\section{Discussion}

The exact pathogenesis of PCME remains unclear; however, the proposed etiological factors include surgical trauma to intraocular tissue, release of prostaglandins, inflammation, vitreous traction on the macula, and ocular hypotony. ${ }^{15,26}$ 
Phacoemulsification uses ultrasonic waves to fragment and emulsify the cataract and in turn generates thermal and mechanical stimuli into the eye, leading to the production of prostaglandins. ${ }^{29}$ These prostaglandins and other inflammatory mediators diffuse posteriorly into the vitreous and disrupt the blood-retinal barrier. Subsequently, the pathological hyperpermeability of retinal blood vessels allows leakage of fluid across the retinal vessel wall, which in turn causes cystic accumulation of fluid in the extracellular spaces of the retina's outer plexiform and inner nuclear layers, resulting in impairment of visual function, despite achieving excellent visual acuity in the early postoperative period. ${ }^{30,31}$

Prefragmentation of the lens with FLs reduces the ultrasound energy/time required for phacoemulsification, and thus it was expected that trauma due to ultrasonic waves and thermal injury due to phacoemulsification would be reduced, causing less release of inflammatory mediators. Contrary to this expectation, eyes that underwent laser pretreatment were found to have higher levels of prostaglandins in the aqueous humor than eyes that underwent manual phacoemulsification. ${ }^{19,27}$ Intraoperative miosis of the pupil observed in some studies following pretreatment with the FL has also been attributed to increased prostaglandin levels after laser treatment. ${ }^{28,32}$ Constricted pupils may increase surgical manipulation during phacoemulsification. Correspondingly, some studies have found a trend of higher risk of PCME development following FLACS compared with manual phacoemulsification. Ewe et $\mathrm{al}^{2}$ found a higher trend of clinical CME development in the FLACS group $(0.8 \%$ of 833 eyes) than the manual phacoemulsification group ( $0.2 \%$ of 458 eyes), as confirmed by OCT imaging in patients presenting with deteriorating visual acuity 3-4 weeks postoperatively compared to previous visits. A subgroup analysis of eyes that had undergone FLACS after speed upgrade to the FL platform revealed that the rate of CME development was higher - 1.05\%. Similarly, Levitz et $\mathrm{al}^{22}$ also reported a trend toward higher rates of clinical CME following FLACS compared with manual phacoemulsification $(1.18 \%$ vs $0.98 \%, p=0.680)$.

Higher rates of CME following FLACS in these studies have been attributed to the increased release of prostaglandins arising from the laser pretreatment. In addition to the inflammatory response generated due to surgical manipulation of intraocular tissue, laser treatment itself has been hypothesized to trigger inflammation. The vaporization of tissue by FLs generates a microplasma of gas and water followed by expanding cavitation bubbles, which have been hypothesized to induce shockwaves in the surrounding ocular structures or alter tissue temperature. ${ }^{27,33}$ Although the exact location of prostaglandin release during laser treatment remains unclear, gas and shockwaves arising during each laser pulse may irritate the ciliary body and trigger prostaglandin release by the unpigmented epithelial layer of the ciliary body. ${ }^{27,34}$ Another potential reason for laserinduced trigger of inflammation could be light-induced toxicity. The near-infrared laser beam used in FLs may get absorbed by pigmented retina and choroid tissue, causing thermal damage during laser treatment. ${ }^{2}$

There have also been reports stating that the risk of CME development is similar following FLACS and CPCS. For instance, Ecsedy et al, ${ }^{16}$ Conrad-Hengerer et al, ${ }^{14}$ Berk et al, ${ }^{35}$ and $\mathrm{Ye}$ et $\mathrm{al}^{20}$ found no significant changes in CSMT (macular thickening) and similar rates of CME development between the two procedures. Nitianandan et al, ${ }^{36}$ in contrast, reported CME to be more prevalent in eyes treated with manual phacoemulsification $(1.1 \%$ vs $0.4 \%)$ than FLACS $(p=0.03)$. This variation in findings of the studies discussed might be due to the interplay of various different factors, including energy used, software versions, and laser settings. Overall duration of the surgery and patient-population demographics and presence/absence of additional risk factors (diabetic retinopathy, cardiovascular diseases, epiretinal membrane, uveitis) may also influence the rate of $\mathrm{CME}$ development after cataract surgery.

Laser-pulse energy is thought to play an important role in the degree of inflammatory response generated by the FL. ${ }^{19,23-25}$ While the cutting process of high pulse energy (microjoule) laser involves expanding cavitation bubbles, it is driven primarily by the plasma itself with low-energy (nanojoule) FLs potentially decreasing the risk of collateral damage to the surrounding ocular tissue and releasing lesser inflammatory mediators. ${ }^{37}$ Low-energy FL use has been reported to produce no meaningful increase in prostaglandin levels in the aqueous humor. ${ }^{38}$ Correspondingly, less pupil constriction with the use of low-energy FLs has been attributed to less release of prostaglandins. ${ }^{28,33}$ Therefore, it can be postulated that low-energy laser platforms may induce low inflammation due to laser pretreatment.

The findings of the present study with the Femto LDV low-energy FL are in alignment with these reports. CSMT change following FLACS and CPCS was found to be comparable. The risk of CME development showed a trend toward lower CME rates in the FLACS group than the CPCS group, whether defined as appearance of cystoid changes near fovea on OCT imaging $(p=0.247)$ or using PREMED study criteria $(p=0.586)$. It is important to 
note that none of the patients in the present study received any topical NSAIDs preoperatively. As it is well documented in the literature that administering preoperative NSAIDs slows the release of inflammatory mediators, including prostaglandins, and reduces intraoperative miosis, it is expected that supplementary topical NSAIDs preoperatively will further improve the results obtained in the present study.

There are a few limitations of this pilot study. There was a significant difference in mean age of patients between the groups, with comparatively younger patients (mean age 68.7 years) in the FLACS group and older (mean age 75.4 years) in the CPCS group. Additional analysis was carried out to check if the difference in age between the groups could confound the findings of the present study. Logistic regression between age and risk of CME revealed an odds ratio of $0.970(p=0.327)$, suggesting that lower age was likely associated with marginally increased risk of developing CME. As such, the lower rate of CME in the low-energy FLACS group (with lower mean age) was an outcome of the difference in the treatment modalities. Additionally, the present study did not directly compare the difference in effective phacoemulsification time between the two groups. In conclusion, FLACS with low-energy FL and CPCS groups were comparable with regard to changes in mean CSMT ( $p=0.902$ ). There were no significant differences in the occurrence of PCME between the FLACS and CPCS groups, although the low-energy FLACS group showed a trend toward lower rates of CME, whether defined using PREMED criteria ( $p=0.586$ ) or OCT imaging showing cystic changes near the fovea $(p=0.247)$. Future studies with larger data sets are required to evaluate the efficacy of low-energy FLs in reducing the risk of postoperative CME.

\section{Disclosure}

The authors report no conflicts of interest for this work.

\section{References}

1. Benitah NR, Arroyo JG. Pseudophakic cystoid macular edema. Int Ophthalmol Clin. 2010;50(1):139-153. doi:10.1097/IIO.0b $013 \mathrm{e} 3181 \mathrm{c} 551 \mathrm{da}$

2. Ewe SY, Oakley CL, Abell RG, Allen PL, Vote BJ. Cystoid macular edema after femtosecond laser-assisted versus phacoemulsification cataract surgery. J Cataract Refract Surg. 2015;41(11):2373-2378. doi:10.1016/j.jcrs.2015.04.031

3. Yonekawa Y, Kim IK. Pseudophakic cystoid macular edema. Curr Opin Ophthalmol. 2012;23(1):26-32. doi:10.1097/ICU.0b013e32834cd5f8

4. Gonzalez-de la Rosa A, Navarro-Partida J, Altamirano-Vallejo JC, et al. Novel triamcinolone acetonide-loaded liposomal topical formulation improves contrast sensitivity outcome after femtosecond laser-assisted cataract surgery. J Ocul Pharmacol Ther. 2019;35 (9):512-521. doi:10.1089/jop.2019.0032
5. Mathys $\mathrm{K}$, Cohen K. Impact of nepafenac $0.1 \%$ on macular thickness and postoperative visual acuity after cataract surgery in patients at low risk for cystoid macular oedema. Eye. 2010;24(1):90-96. doi:10.1038/eye.2009.10

6. Wang J, Sramek C, Paulus YM, et al. Retinal safety of near-infrared lasers in cataract surgery. J Biomed Opt. 2012;17(9):95001. doi:10.1117/1.JBO.17.9.095001

7. Abell RG, Kerr NM, Vote BJ. Toward zero effective phacoemulsification time using femtosecond laser pretreatment. Ophthalmology. 2013;120(5):942-948. doi:10.1016/j.ophtha.2012.11.045

8. Abell RG, Kerr NM, Vote BJ. Femtosecond laser-assisted cataract surgery compared with conventional cataract surgery. Clin Exp Ophthalmol. 2013;41(5):455-462. doi:10.1111/ceo.12025

9. Conrad-Hengerer I, Al Juburi M, Schultz T, Hengerer FH, Dick HB. Corneal endothelial cell loss and corneal thickness in conventional compared with femtosecond laser-assisted cataract surgery: three-month follow-up. $J$ Cataract Refract Surg. 2013;39 (9):1307-1313. doi:10.1016/j.jcrs.2013.05.033

10. Conrad-Hengerer I, Hengerer FH, Schultz T, Dick HB. Effect of femtosecond laser fragmentation on effective phacoemulsification time in cataract surgery. $J$ Refract Surg. 2012;28(12):879-883. doi:10.3928/1081597X-20121116-02

11. Reddy KP, Kandulla J, Auffarth GU. Effectiveness and safety of femtosecond laser-assisted lens fragmentation and anterior capsulotomy versus the manual technique in cataract surgery. J Cataract Refract Surg. 2013;39(9):1297-1306. doi:10.1016/j. jcrs.2013.05.035

12. He L, Sheehy K, Culbertson W. Femtosecond laser-assisted cataract surgery. Curr Opin Ophthalmol. 2011;22(1):43-52. doi:10.1097/ ICU.0b013e3283414f76

13. Abell RG, Allen PL, Vote BJ. Anterior chamber flare after femtosecond laser-assisted cataract surgery. J Cataract Refract Surg. 2013;39 (9):1321-1326. doi:10.1016/j.jcrs.2013.06.009

14. Conrad-Hengerer I, Hengerer FH, Al Juburi M, Schultz T, Dick HB. Femtosecond laser-induced macular changes and anterior segment inflammation in cataract surgery. $J$ Refract Surg. 2014;30 (4):222-226. doi:10.3928/1081597X-20140321-01

15. Nagy ZZ, Ecsedy M, Kovacs I, et al. Macular morphology assessed by optical coherence tomography image segmentation after femtosecond laser-assisted and standard cataract surgery. $J$ Cataract Refract Surg. 2012;38(6):941-946. doi:10.1016/j. jcrs.2012.02.031

16. Ecsedy M, Mihaltz K, Kovacs I, Takacs A, Filkorn T, Nagy ZZ. Effect of femtosecond laser cataract surgery on the macula. $J$ Refract Surg. 2011;27(10):717-722. doi:10.3928/1081597X-20110825-01

17. Grzybowski A, Sikorski BL, Ascaso FJ, Huerva V. Pseudophakic cystoid macular edema: update 2016. Clin Interv Aging. 2016;11:1221-1229. doi:10.2147/CIA.S111761

18. Roberts HW, Wagh VK, Sullivan DL, et al. A randomized controlled trial comparing femtosecond laser-assisted cataract surgery versus conventional phacoemulsification surgery. J Cataract Refract Surg. 2019;45(1):11-20. doi:10.1016/j.jcrs.2018.08.033

19. Schultz T, Joachim SC, Kuehn M, Dick HB. Changes in prostaglandin levels in patients undergoing femtosecond laser-assisted cataract surgery. $J$ Refract Surg. 2013;29(11):742-747. doi:10.3928/ 1081597X-20131021-03

20. Ye Z, Li Z, He S. A meta-analysis comparing postoperative complications and outcomes of femtosecond laser-assisted cataract surgery versus conventional phacoemulsification for cataract. J Ophthalmol. 2017;2017:3849152. doi:10.1155/2017/3849152

21. Gama I. Pseudophakic macular edema (irvine-gass syndrome): has the treatment changed? MJ Opht. 2016;1(1):004.

22. Levitz L, Reich J, Roberts TV, Lawless M. Incidence of cystoid macular edema: femtosecond laser-assisted cataract surgery versus manual cataract surgery. J Cataract Refract Surg. 2015;41 (3):683-686. doi:10.1016/j.jcrs.2014.11.039 
23. Chen $\mathrm{H}$, Lin $\mathrm{H}$, Chen $\mathrm{W}$, et al. Topical $0.1 \%$ bromfenac sodium for intraoperative miosis prevention and prostaglandin E2 inhibition in femtosecond laser-assisted cataract surgery. J Ocul Pharmacol Ther. 2017;33(3):193-201. doi:10.1089/jop.2016.0114

24. Jun JH, Yoo YS, Lim SA, Joo CK. Effects of topical ketorolac tromethamine $0.45 \%$ on intraoperative miosis and prostaglandin E2 release during femtosecond laser-assisted cataract surgery. J Cataract Refract Surg. 2017;43(4):492-497. doi:10.1016/j.jcrs.2017.01.011

25. Liu YC, Setiawan M, Ang M, Yam GHF, Mehta JS. Changes in aqueous oxidative stress, prostaglandins, and cytokines: comparisons of low-energy femtosecond laser-assisted cataract surgery versus conventional phacoemulsification. J Cataract Refract Surg. 2019;45 (2):196-203. doi:10.1016/j.jcrs.2018.09.022

26. Asena BS, Karahan E, Kaskaloglu M. Retinal and choroidal thickness after femtosecond laser-assisted and standard phacoemulsification. Clin Ophthalmol. 2017;11:1541-1547. doi:10.2147/OPTH.S127792

27. Schultz T, Joachim SC, Stellbogen M, Dick HB. Prostaglandin release during femtosecond laser-assisted cataract surgery: main inducer. J Refract Surg. 2015;31(2):78-81. doi:10.3928/1081597X20150122-01

28. Mirshahi A, Ap K. Changes in pupil area during low-energy femtosecond laser-assisted cataract surgery. J Ophthalmic Vis Res. 2019;14 (3):251-256. doi:10.18502/jovr.v14i3.4780

29. Dick HB, Schultz T. A review of laser-assisted versus traditional phacoemulsification cataract surgery. Ophthalmol Ther. 2017;6 (1):7-18. doi:10.1007/s40123-017-0080-z

30. Jittpoonkuson T, Garcia PM, Rosen RB. Correlation between fluorescein angiography and spectral-domain optical coherence tomography in the diagnosis of cystoid macular edema. Br J Ophthalmol. 2010;94(9):1197-1200. doi:10.1136/bjo.2009.170589

31. Walter KA, Lee RY, Chen K, Komanski C. Incidence of cystoid macular edema following routine cataract surgery using NSAIDs alone or with corticosteroids. Arq Bras Oftalmol. 2019. AHEAD.
32. Dick HB, Gerste RD, Schultz T. Laser cataract surgery: curse of the small pupil. J Refract Surg. 2013;29(10):662. doi:10.3928/ 1081597X-20130920-01

33. Jun JH, Hwang KY, Chang SD, Joo C-K. Pupil-size alterations induced by photodisruption during femtosecond laser-assisted cataract surgery. $J$ Cataract Refract Surg. 2015;41(2):278-285. doi:10.1016/j.jcrs.2014.10.027

34. Schultz T, Joachim SC, Szuler M, Stellbogen M, Dick HB. NSAID pretreatment inhibits prostaglandin release in femtosecond laser-assisted cataract surgery. $J$ Refract Surg. 2015;31 (12):791-794. doi:10.3928/1081597X-20151111-01

35. Berk TA, Schlenker MB, Campos-Moller X, Pereira AM, Ahmed IIK. Visual and refractive outcomes in manual versus femtosecond laser-assisted cataract surgery: a single-center retrospective cohort analysis of 1838 eyes. Ophthalmology. 2018;125 (8):1172-1180. doi:10.1016/j.ophtha.2018.01.028

36. Nithianandan $H$, Jegatheeswaran V, Dalal V, et al. Refractive laser-assisted cataract surgery versus conventional manual surgery: comparing efficacy and safety in 3144 eyes. Am J Ophthalmol. 2019;206:32-39. doi:10.1016/j.ajo.2019.04.010

37. Pepose J, Lubatschowski H. Comparing femtosecond lasers. Cataract \& Refractive Surgery Today. 2008;10:45-51.

38. Schwarzenbacher L, Schartmueller D, Leydolt C, Menapace R. Intraindividual comparison of proinflammatory cytokines (IL-1 $\beta$, IL-6) and total-prostaglandin (PG) following femtosecond laserassisted cataract surgery using a low-energy, high-frequency femtosecond laser-device compared to manual cataract surgery. Invest Ophthalmol Vis Sci. 2018;59(9):4789.
Clinical Ophthalmology

\section{Publish your work in this journal}

Clinical Ophthalmology is an international, peer-reviewed journal covering all subspecialties within ophthalmology. Key topics include: Optometry; Visual science; Pharmacology and drug therapy in eye diseases; Basic Sciences; Primary and Secondary eye care; Patient Safety and Quality of Care Improvements. This journal is indexed on PubMed

\section{Dovepress}

Central and CAS, and is the official journal of The Society of Clinical Ophthalmology (SCO). The manuscript management system is completely online and includes a very quick and fair peer-review system, which is all easy to use. Visit http://www.dovepress.com/ testimonials.php to read real quotes from published authors. 